\title{
LncRNAs expression profile in peripheral blood mononuclear cells from multiple sclerosis patients
}

\author{
Chiara Fenoglio ${ }^{\mathrm{a}, *, 1}$, Emanuela Oldoni ${ }^{\mathrm{a}, \mathrm{b}, 1}$, Maria Serpente ${ }^{\mathrm{a}}$, Milena A. De Riz ${ }^{\mathrm{c}}$, Marina Arcaro ${ }^{\mathrm{c}}$, \\ Marianna D'Anca $^{\mathrm{a}}$, Anna M. Pietroboni ${ }^{\mathrm{c}}$, Alberto Calvi ${ }^{\mathrm{a}}$, Elisabetta Lecchi ${ }^{\mathrm{c}}$, An Goris ${ }^{\mathrm{b}}$, \\ Klara Mallants $^{\mathrm{b}}$, Bénédicte Dubois ${ }^{\mathrm{b}, \mathrm{d}}$, Cristoforo Comi ${ }^{\mathrm{e}}$, Roberto Cantello ${ }^{\mathrm{e}}$, Elio Scarpini ${ }^{\mathrm{a}, \mathrm{c}}$, \\ Daniela Galimberti ${ }^{\mathrm{a}, \mathrm{c}}$

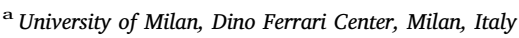 \\ b Laboratory for Neuroimmunology, Department of Neurosciences, KU Leuven, Herestraat 49 bus 1022, 3000 Leuven, Belgium \\ ${ }^{\mathrm{c}}$ Fondazione IRCCS Cà Granda Ospedale Maggiore Policlinico, Neurodegenerative Diseases Unit, Milan, Italy \\ ${ }^{\mathrm{d}}$ Department of Neurology, University Hospitals Leuven, Herestraat 49, 3000 Leuven, Belgium \\ ${ }^{\mathrm{e}}$ Department of Translational Medicine, Section of Neurology, University of Eastern Piedmont Amedeo Avogadro, Novara, Italy
}

\section{A R T I C L E I N F O}

\section{Keywords:}

Multiple Sclerosis

Non coding RNA

Gene expression

Biomarker

\begin{abstract}
A B S T R A C T
LncRNA PCR arrays containing 90 common LncRNAs were used to screen lncRNA expression levels in PBMC from a discovery population of patients with MS. Data from discovery and replications cohorts showed a generalized dysregulation of lncRNA levels in MS patients compared with controls. MALAT1, MEG9, NRON, ANRIL, TUG1, XIST, SOX2OT, GOMAFU, HULC, BACE-1AS were significantly downregulated in MS patients in comparison with controls. Therefore, we performed a validation analysis in an independent cohort of Belgian origin. In this study, NRON and TUG1 downregulations in MS patients compared with controls were confirmed ( $p \leq .05$ and $p \leq .0001$ respectively), whereas considering the other lncRNAs, the statistical threshold was not reached. LncRNAs profiling could thus represent a new challenge in the research of easy detectable biomarkers of disease susceptibility and progression.
\end{abstract}

\section{Introduction}

Multiple Sclerosis (MS) is a chronic complex autoimmune disease of the Central Nervous System (CNS) characterized by demyelination and axonal degeneration. Indeed, ethnic, environmental and genetic factors are involved in the initiation and progression of MS.

Clinically, different MS subtypes have been described (relapsingremitting -RR, secondary progressive -SP, and primary progressive - $\mathrm{PP}$ ) and within each subtype a considerable individual variation in disease course could occur. PPMS likely has a different pathogenesis than RRMS in which inflammatory events seem to have a pivotal role (McFarland and Martin, 2007). In PPMS, neurodegeneration is likely prominent, as shown by the development of motor disability, cognitive deficits and brain atrophy (Trapp and Nave, 2008).
At present, no laboratory measure that reliably correlates with or predicts disease activity and response to therapies exists. In the last few years, several evidence supporting a key role of epigenetic changes in MS pathogenesis has been highlighted (Zhang and Zhang, 2015). Among these, non coding RNAs (ncRNAs) are involved in neural development and in the progression of neurodegenerative (Wan et al., 2017)(Zhou and Xu, 2015)(Lourenco et al., 2015) and demyelinating diseases ( $\mathrm{Wu}$ et al., 2013). Moreover, they have been largely investigated as potential biomarkers of disease activity (Zhang et al., 2016)(Santoro et al., 2016).

Long non coding RNAs (lncRNAs) are transcripts with a low or no protein-coding potential, with a length of $>200$ nucleotides. These non-coding RNA molecules share some common features with microRNAs (miRNA) as they could be spliced, capped and

\footnotetext{
* Corresponding author.

E-mail addresses: chiara.fenoglio@unimi.it (C. Fenoglio), emanuela.oldoni@kuleuven.be (E. Oldoni), maria.serpente@unimi.it (M. Serpente), milena.deriz@policlinico.mi.it (M.A. De Riz), marina.arcaro@unimi.it (M. Arcaro), marianna.danca@unimi.it (M. D'Anca),

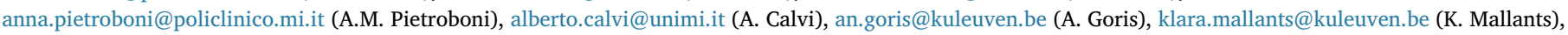
benedicte.dubois@kuleuven.be (B. Dubois), cristoforo.comi@med.uniupo.it (C. Comi), roberto.cantello@med.unipo.it (R. Cantello), elio.scarpini@unimi.it (E. Scarpini), daniela.galimberti@unimi.it (D. Galimberti).

${ }^{1}$ Authors contributed equally to the work.
} 
polyadenylated (Shi et al., 2013). They regulate gene expression at the epigenetic, transcriptional, and post-transcriptional level in cellular homeostasis (Wan et al., 2017).

Interestingly, a growing body of evidence demonstrates that lncRNAs get dragged into different cells pathway, as for example in the regulation of immune cells activity. About this issue, specific expression pattern in lymphocytes lineages have been described (Aune et al., 2016). Since it is known that these cells are involved in the pathogenesis of MS, IncRNAs may control important steps of their imbalanced activity (Kleinewietfeld and Hafler, 2014)(Sinha et al., 2015), suggesting that they could play a role in the progression of the disease.

Recently, three lncRNAs have been identified as deregulated in serum of RRMS patients compared to controls: nuclear paraspeckle assembly transcript 1 (NEAT1), taurine up-regulated 1 (TUG1), and 7SK small nuclear (RN7SK RNA)(Santoro et al., 2016). Conversely, Zhang et al. (2016) identified a subset of dysregulated lncRNAs in peripheral blood mononuclear cells (PBMC), by microarrays analysis, in a population consisting of 26 MS patients. Furthermore, significantly dysregulated levels of Plasmacytoma variant translocation 1 (PVT1), Fas cell surface death receptor-antisense 1 (FAS-AS1) e and Heterogeneous nuclear ribonucleoprotein $L$ (THRIL) in RRMS patients compared with controls were found (Eftekharian et al., 2017).

The lncRNA growth arrest special 5, GAS5, was identified as epigenetic regulator of microglial polarization and its role in the attenuation of the progression of experimental autoimmune encephalomyelitis (EAE) and in the promotion of remyelination was shown (Sun et al., 2017). Lastly, the lncRNA HOX transcript antisense intergenic RNA (HOTAIR) has been found to be involved in the pathogenesis of EAE (Pahlevan Kakhki et al., 2017).

Given these premises, we investigated the role of lncRNA isolated from PBMC in MS patients by using a comprehensive methodological approach. To this purpose, we initially performed and explorative analysis in 10 patients, by using specific arrays that enable to screening simultaneously 90 lncRNAs involved in autoimmunity and human inflammatory response, in order to identify those dysregulated. The further step was aimed to validate best hits IncRNAs in a larger population composed by 30 cases and 25 controls, with specific Taqman probes for the quantitative real-time PCR assay. Lastly, a replication step was performed in an independent Belgian cohort consisting of 24 cases and 23 controls, by droplet digital PCR system.

\section{Materials and methods}

\subsection{Population and sample collection}

The Italian cohort was recruited at the Multiple Sclerosis Center of the Cà Granda Foundation IRCCS Ospedale Maggiore Policlinico of Milan and at the Department of Translational Medicine, Section of Neurology of the University of Eastern Piedmont, Novara, while Belgian patients came from the University Hospital of Leuven.

Patients underwent the standard workup for MS, including medical history, physical and neurological examination, screening laboratory tests, brain Magnetic Resonance Imaging. Diagnoses met current consensus criteria (Polman et al., 2011).

None of MS patients was treated with immunomodulatory therapies at the time of blood sampling, as well as steroid. Moreover, patients with other autoimmune diseases, oncologic diseases or chronic infectious diseases were excluded.

In order to reduce any potential confounding factors, blood withdrawal was performed early in the morning between 8.00 and $10.00 \mathrm{am}$, and RR patients were not in an acute phase. The control group involved healthy subjects, matched for age, gender and ethnic background. Characteristics of the population are summarized in Table 1.
Table 1

Characteristics of populations. $\mathrm{RR}=$ relapsing-remitting; $\mathrm{PP}=$ primary progressive; EDSS $=$ Expanded disability status scale.

\begin{tabular}{|c|c|c|c|}
\hline & Controls & RR-MS & PP-MS \\
\hline \multicolumn{4}{|l|}{ Italian population } \\
\hline $\mathrm{N}$ & 31 & 27 & 13 \\
\hline Gender (M:F) & $15: 16$ & $12: 15$ & $5: 8$ \\
\hline Mean age, years $\pm S D$ & $34,7 \pm 7,8$ & $35,9 \pm 1,7$ & $45,5 \pm 2,0$ \\
\hline Mean age at onset, years \pm SD & NA & $35,1 \pm 7,0$ & $41,2 \pm 7,3$ \\
\hline Mean EDSS (range) & NA & $2,5(0-5)$ & $6(3-7)$ \\
\hline \multicolumn{4}{|l|}{ Belgian population } \\
\hline $\mathrm{N}$ & 23 & 17 & 7 \\
\hline Gender (M:F) & $11: 12$ & $8: 9$ & $3: 4$ \\
\hline Mean age, years $\pm S D$ & $45,5 \pm 9,0$ & $36,6 \pm 11,6$ & $52,8 \pm 5,2$ \\
\hline Mean age at onset, years \pm SD & NA & $36,9 \pm 10,0$ & $45,8 \pm 5,1$ \\
\hline Mean EDSS (range) & NA & $3,5(0-4)$ & $6(2,5-6,5)$ \\
\hline
\end{tabular}

\subsection{IncRNA extraction from $P B M C$}

Total RNA from PBMC was extracted using phenol-chloroform protocol, which enables purification of total RNA. Quantity and purity was evaluated by Nanodrop ${ }^{\mathrm{TM}}$ Lite Spectrophotometer (Thermo Fisher Scientific). RNA purity was measured by optical density and only samples with an OD 260/280 ratio ranging from 1.8 to 2 and an OD $260 / 230$ of 1.8 or greater were used.

\subsection{IncRNA expression levels}

\subsubsection{Explorative analysis}

The explorative analysis was carried out on 10 MS patients (5 RRMS and 5 PP-MS) and 6 controls randomly chosen from the Italian cohort. LncRNAs were retro-transcribed using cDNA Synthesis First Strand kit (Qiagen) for use with SYBR green technology arrays. LncRNAs expression levels were evaluated by human LncProfiler qPCR array (System Biosciences, SBI), a panel for the quantification of 90 different lncRNAs related by pathway or disease, in SYBR green Technology according to the instructions of the manufacturer using ABI 7500 Fast Real Time System (Applied BioSystems, ABI) and the normalization was performed using five housekeeping genes (Fig. 1A).

\subsubsection{Validation analysis and NFATc expression levels}

Total RNA (including LncRNAs) was retro-transcribed using SuperScript III Reverse Transcriptase (Life Technologies) that provides increased specificity with gene-specific primers (GSPs).

Customized plates with TaqMan probes were drawn for best hits and lncRNAs expression levels were measured in RT-PCR by ABI 7500 fast Real time PCR System (Applied BioSystems). The TaqMan gene expression assay Hs00542675_m1 was used to test the expression level of NFATc gene, target gene of NRON, in the validation population. Two housekeeping genes, 18S and GAPDH, were used to provide normalization.

\subsubsection{Replication analysis}

LncRNAs were retro-transcribed using Multiscribe Reverse Transcriptase (Thermo Fisher Scientific) and their expression levels were measured by droplet digital PCR (QX200 ddPCR BioRad), using Taqman Probes. Three different housekeeping genes were used to provide the normalization: 18S, POLR2A and GAPDH.

\subsection{Statistical analysis}

GraphPad Prism 6.0 software and $\mathrm{R}$ were used for statistical analysis. Test of normality was performed by using the Shapiro-Wilk's test. Subsequently, comparisons of lncRNA levels, expressed by relative quantification (RQ), between patients and controls were performed by using non parametric Kruskall-Wallis test and the correction for 
A)

\begin{tabular}{|c|c|c|c|c|c|c|c|c|c|c|c|c|}
\hline & 1 & 2 & 3 & 4 & 5 & 6 & 7 & 8 & 9 & 10 & 11 & 12 \\
\hline A & $21 \mathrm{~A}$ & 7SK & 7SL & Air & AK023948 & Alpha 280 & Alpha 250 & ANRIL & anti-NOS2A & antiPeg11 & BACE1AS & BC200 \\
\hline B & $\begin{array}{c}\text { CAR } \\
\text { Intergenic }\end{array}$ & $\begin{array}{c}\text { DHFR } \\
\text { upstream }\end{array}$ & Dio3os & DISC2 & DLG2AS & $\begin{array}{c}\text { E2F4 } \\
\text { antisense }\end{array}$ & EgoA & EGO B & Emx2os & $\begin{array}{c}\text { Evf1 and } \\
\text { EVF2 }\end{array}$ & GAS5-family & Gomafu \\
\hline C & H19 & $\begin{array}{c}\text { H19 } \\
\text { antisense }\end{array}$ & $\begin{array}{c}\text { H19 } \\
\text { upstream }\end{array}$ & HAR1A & HAR1B & HOTAIR & HOTAIRM1 & HOTTIP & Hoxa11as & HOXA3as & HOXA6as & HULC \\
\hline D & IGF2AS & IPW & Jpx & Kcnq1ot1 & KRASP1 & L1PA16 & p21 & RoR & SFMBT2 & VLDLR & LOC285194 & LUST \\
\hline & Malat1 & mascRNA & MEG3 & MEG9 & MER11C & nCR-UPAR & NDM29 & NEAT1 & Nespas & NRON & NTT & p53 mRNA \\
\hline$F$ & PCGEM1 & $\begin{array}{c}\text { PR } \\
\text { antisense }\end{array}$ & PRINS & $\begin{array}{c}\text { PSF } \\
\text { inhibiting }\end{array}$ & PTENP1 & RNCR3 & SAF & SCA8 & snaR & SNHG1 & SNHG3 & SNHG 4 \\
\hline 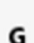 & SNHGS & SNHG6 & Sox2ot & SRA & ST7OT & TEA ncRNAs & Tmevpg1 & TncRNA & Tsix & TUG1 & UCA1 & UM9-5 \\
\hline & WT1-AS & Xist & YRNA-1 & Zeb2NAT & Zfas1 & Zfh $\times 2$ as & 185 rRNA & RNU43 & GAPDH & LAMIN A/C & Human U6 & $\begin{array}{l}\text { No assay } \\
\text { control }\end{array}$ \\
\hline
\end{tabular}

B)

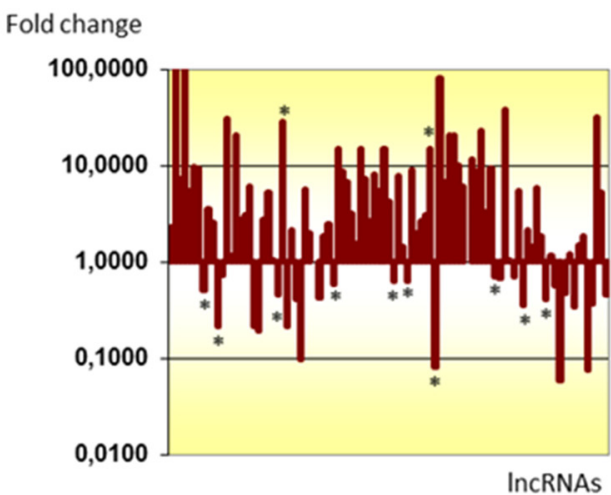

A)
MS

IncRNAs
Fold change 100,0000

10,0000
1,0000
0,1000
0,0100

B)
RR-MS

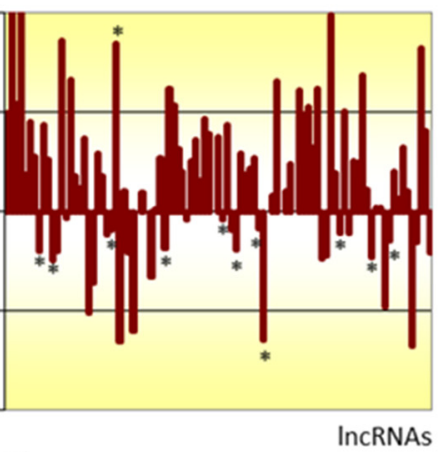

IncRNAs

PP-MS

Fold change

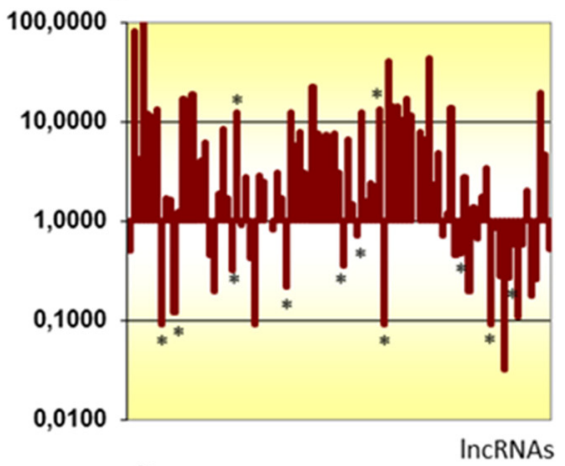

C)

IncRNAs

Fig. 1. (A) Scheme of the human LncProfiler $q P C R$ array (SBI). (B) LncRNAs expression levels from human lncProfiler array. Each bar represents a lncRNA according to the order of table in Fig. 1A. A) MS patients $(n=10)$ vs controls $(n=6)$. B) RRMS $(n=5=$ vs controls. C) PPMS patients $(n=5)$ vs controls. Statistical significant lncRNA dysregulations have been calculated by non parametric Kruskall-Wallis test post-hoc Dunn's test and marked with*p $\leq .05$. Test of normality: Shapiro-Wilk's test.

multiple comparisons was performed using the post-hoc Dunn's test.

Lastly, for the analysis of the replication population data, the Poisson statistical analysis of the numbers of positive and negative droplets yields absolute quantitation of the target sequence. The analysis, in this case, was performed by QuantaSoft software (BioRad), while R was used to compare cases and controls and disease forms.

Correlation analysis with clinical data such as disease duration and Expanded Disability Status Scale (EDSS) score were performed using Spearman test.

\section{Results}

\subsection{Explorative analysis}

LncRNAs expression profiling performed by lncProfiler array showed a generalized dysregulation in the overall MS population compared with controls (Fig. 1B) and also after stratification according to disease subtype (Fig. 1B-1C). Twelve out of the 90 lncRNAs analyzed were nominally significant $(p<.05)$ and underwent the further validation step.

\subsection{Validation analysis}

Results were subsequently validated in the Italian whole cohort (Table 1) and 10 out of 12 lncRNAs were significantly down-regulated in MS patients compared to controls (Fig. $2 \mathrm{~A}$ and B). In particular,
MALAT-1, NRON and MEG-9 were found to be significantly downregulated in MS patiens compared with controls $(p<.05)$ whereas ANRIL, TUG1, XIST $(p<.0001)$ and SOX2OT $(p<.001)$ were strongly down-regulated also in RR-MS. Lastly, GOMAFU, HULC $(\mathrm{p}<.0001)$ and BACE-1AS $(\mathrm{p}<.001)$ showed a robust down-regulation both in RR-MS and PP-MS in comparison with controls.

The expression level of NFATc, the NRON target gene, was also determined. No significant differences were found when comparing NFATc expression levels in patients and controls $(\mathrm{p}<.05)$ and no correlations have been found among NRON and NFATc expression levels as well $(r=0.18, p=.34)$.

Correlations between lncRNA expression levels and clinical variables such as disease duration and EDSS score were tested. ANRIL and TUG1 correlated with disability expressed by EDSS ( $p=.024 r=0.576$ and $p=.007 r=0.434$ respectively, Figs. 3 and 4 ), whereas NRON showed a correlation with disease duration $(p=.05$ and $r=0.528$, Fig. 4).

\subsection{Replication analysis}

Among lncRNAs that passed the validation analysis, NRON, TUG1 and GOMAFU underwent the further replication step in an independent Belgian cohort (Table 1), using a different technology, the digital droplet PCR (ddPCR). Among all the lncRNAs previously validated, they were specifically chosen for the replication analysis because of their biologic meaning and relation with the disease. 

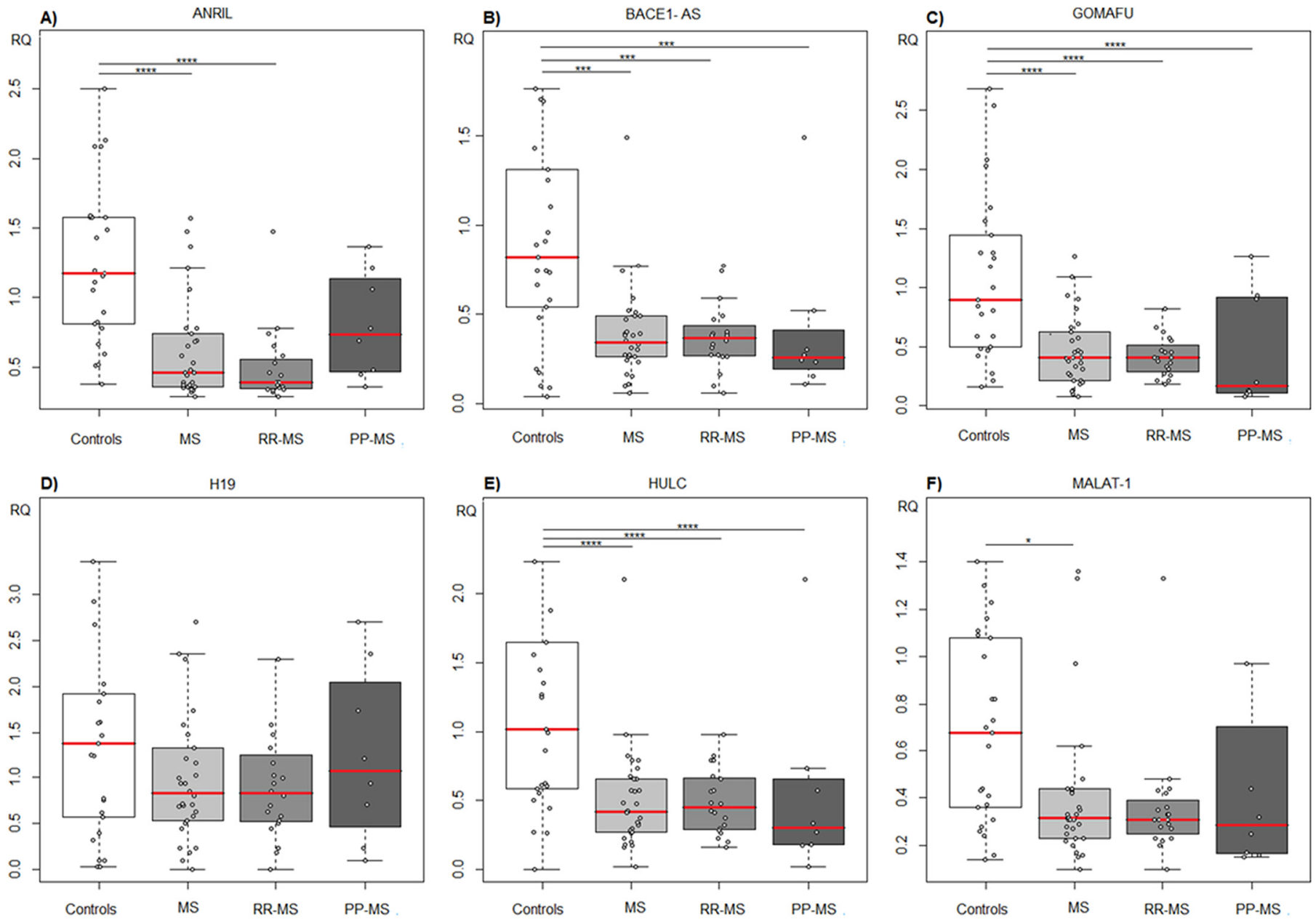

Fig. 2. panels A-L: Validation analysis. LncRNAs expression levels obtained by RT-PCR in the Italian cohort, are relative and expressed as relative quantification (RQ) (mean $\pm \mathrm{SD}$ ) and stratified by disease forms (RR = relapsing-remitting and progressive). GAPDH and 18S were used as normalizer genes. Each dot represents one subject. Median value for each group is indicated in red. Test of normality: Shapiro-Wilk's test. ${ }^{*} p<.05,{ }^{* *} p=.01{ }^{* * *} p<.001 * * * * p<.0001$ calculated by non parametric Kruskall-Wallis test post-hoc Dunn's test.

We confirmed that NRON and TUG1 had lower levels in MS patients compared with controls ( $\mathrm{p}<.05$ and $\mathrm{p}<.0001$ respectively). In particular, TUG1 was dysregulated both in RR and PP form. In contrast, the replication study failed for GOMAFU although a trend toward a downregulation of its expression levels emerged in MS cases compared to controls (Fig. 5).

Correlations between EDSS score and lncRNAs expression levels failed to be validated although a trend toward a positive correlation between NRON level with disease duration was found $(p=.048$ $r=0.478$, Fig. 6).

\section{Discussion}

Herein, we showed a specific signature of lncRNAs dysregulated in MS patients compared with controls. Emerging evidence revealed that lncRNAs play a pivotal role in the regulation of immunological functions and autoimmunity (Wu et al., 2015). Nevertheless, since there is not, at present, a clear pathophysiological hypothesis concerning the possible role of a specific lncRNA profile in MS, we chose to analyze the most investigated lncRNAs in inflammatory diseases, through a commercial array. In order to highlight the role of these molecules in MS, we deeply investigated IncRNAs expression profile in different independent cohorts of MS patients using different technologies.

The results showed a general dysregulation of lncRNAs expression levels in MS patients compared with controls in the explorative analysis cohort, independent of the MS subtype. The further validation step performed in the Italian MS and control population confirmed the dysregulations observed in the explorative initial step.

Among the lncRNAs validated, we further focused on NRON, TUG1 and GOMAFU for the subsequent replication step in the Belgian cohort. These specific lncRNAs were chosen because of their possible implication in MS pathogenesis due to their involvement in inflammatory and neurodegenerative processes. NRON and TUG1 were replicated in the Belgian cohort whereas GOMAFU didn't pass the replication step although a trend toward a downregulation of its expression level in patients was found.

NRON is a lncRNA repressor of the nuclear factor of activated T cells (NFAT), which interacts with members of the importin-beta superfamily and acts as specific regulator of NFAT nuclear trafficking (Turner et al., 2014). NFATs regulate the transcriptional induction of genes encoding for immune modulators/activators such as granulocyte-macrophage colony-stimulating factor (GM-CSF), gamma interferon (IFN $\gamma$ ), CD5, CD25, CD28, CD40, interleukin- (IL-) 2, IL-3, IL-4, IL-5, IL-13, IL8(Kipanyula et al., 2016). In T lymphocytes, NFAT proteins govern gene expression that regulates their development, activation, differentiation, as well as the induction and maintenance of T-cell tolerance. Furthermore, data from murine and cell lines models highlight the role of NFAT $/ \mathrm{Ca}^{2+}$ calmodulin pathway in CNS. Dietz et al. demonstrated, also, that NFAT1 and NFAT2 deficit attenuate EAE in animal model, underlying the importance of this pathway in pathogenesis of the MS 

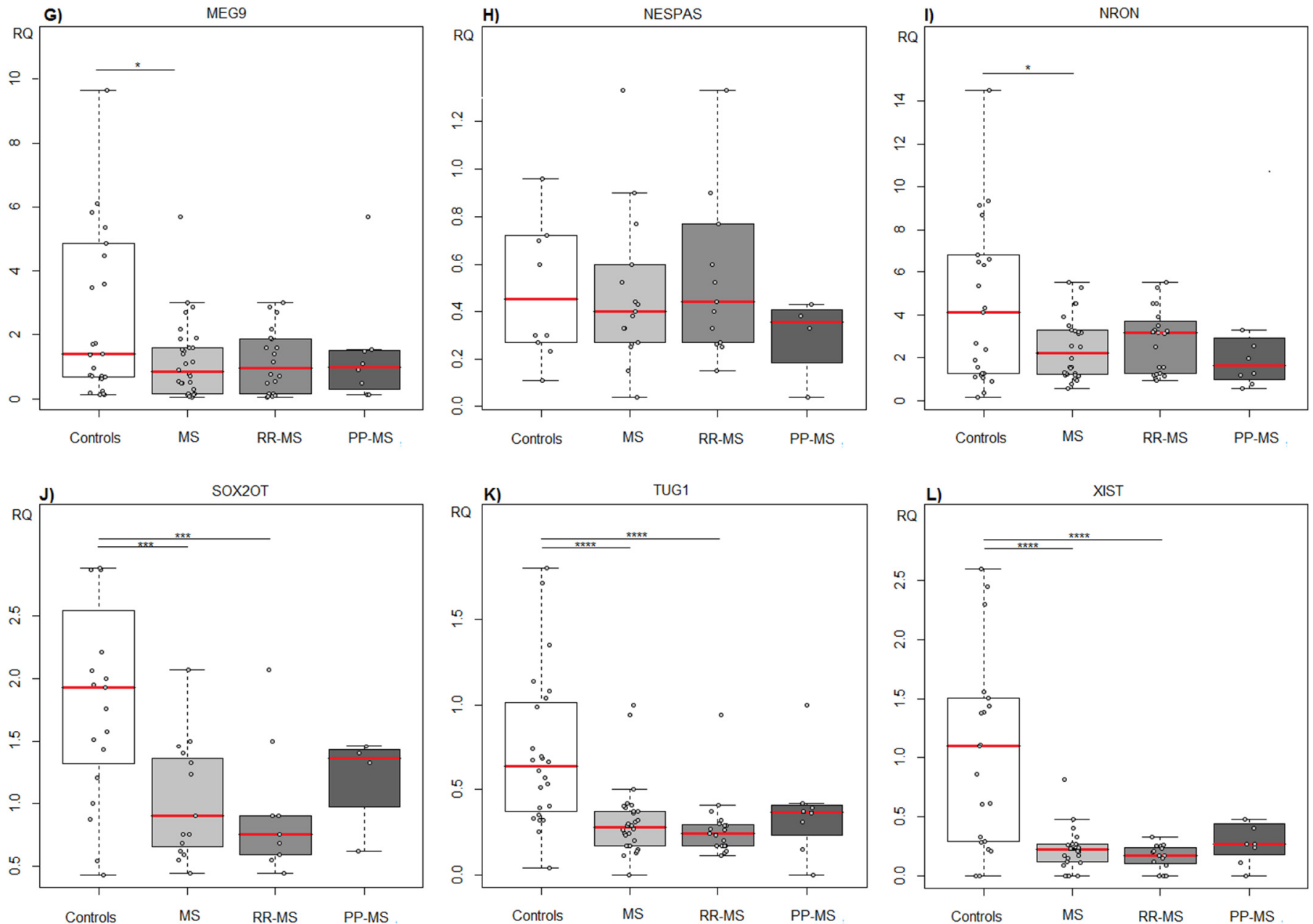

Fig. 2. (continued)

disease (Dietz et al., 2015). NRON expression level in PBMC of MS patients was found to be lower in cases compared with controls. Therefore, we investigated the expression level of its target gene NFAT, under the hypothesis of a regulation of NFAT expression orchestrated by NRON. However, we didn't find any significant differences in NFAT expression level in MS cases compared with controls and no correlation was found with NRON expression level as well. NRON/NFAT pathway is a complex, then, probably, other alternative mechanisms could drive its regulation. TUG1 is expressed in the developing retina and brain, showing the highest levels in the cortex. It is involved in the regulation of cell cycle and apoptotic processes mediated by p53 (Guttman et al., 2011) Indeed, TUG1 expression is activated after DNA damage, and its promoter presents several p53 binding sites. When it is associated with polycomb repressive complex 2 (PRC2), it acts as repressor for a number of cell cycle genes (Khalil et al., 2009) Moreover, in the neurodegenerative disease context, it was found deregulated in patients suffering from the trinucleotide repeat neurodegenerative conditions (Johnson, 2012).

A robust down-regulation of TUG1 expression levels was observed in MS patient compared with controls. Interestingly, stratifying the results according to the disease form, the lowest expression levels were observed in PPMS. TUG1, shown to be involved in the apoptotic-p53 pathway, could play a key role in MS disease progression (Rossi et al., 2014).

In contrast with our results, Santoro et al. showed an opposite trend of TUG1 expression, in the serum from MS patients (Santoro et al., 2016). The reason for such discrepancy could be related to the different biological source chosen for the analysis or to a possible active role of
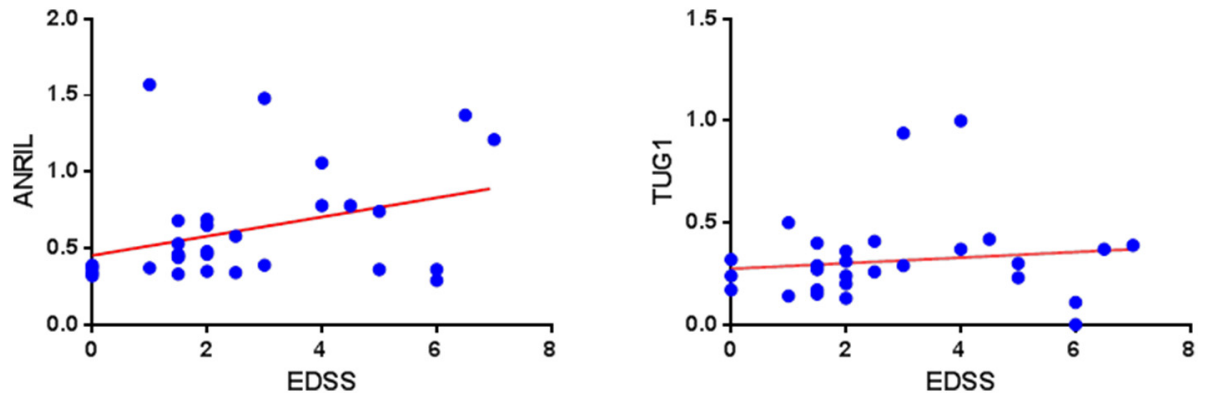

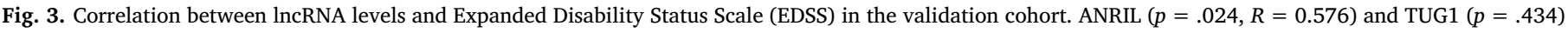
expression levels correlated with EDSS. Correlation was calculated by Spearman's test. 


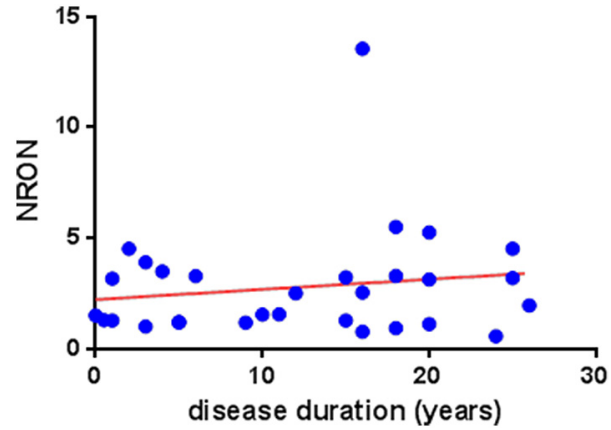

Fig. 4. Correlation between NRON levels and disease duration (years) in the validation cohort. $P=.05, R=0.528$.

TUG1 in the intercellular communication. Its increased levels as free circulating could originate from the release in the cellular milieu by exosomes, an enriched source of non-coding RNA.

GOMAFU, alias MIAT, is predominantly expressed in CNS (Mercer et al., 2008), where regulates the differentiation of neural stem cells into oligodendrocytes (Mercer et al., 2010). GOMAFU can bind the splicing factor 1 (SF1) protein through its UACUAAC repeat sequences. This sequence is a much stronger intron branch point sequence than found in most mammalian introns. Moreover, in vitro GOMAFU binding to SF1 can inhibit splicing and spliceosomal complex formation suggesting it can function to regulate splicing efficiency (Tsuiji et al., 2011). Dysregulation of GOMAFU leads to alternative splicing patterns that resemble those observed in schizophrenia for the archetypal schizophrenia-associated genes DISC1 and ERBB4 (Barry et al., 2014)

Although in the Italian cohort GOMAFU showed a strong downregulation in MS patients compared to controls, including in RRMS and PPMS forms, we didn't replicate the results in the Belgian population and probably this could be due to the small number of patients.

Moreover, it's interesting to notice that NRON correlates with the disease duration, while ANRIL and TUG1 positively correlate with the disability measured by EDSS. This last aspect can be related to the progression of neurodegeneration. The association between ncRNAs and EDSS as well as disease duration has been previously reported (Gandhi et al., 2013)(Fenoglio et al., 2013)

\section{Conclusions}

Herein, we described the investigation of the expression profile of

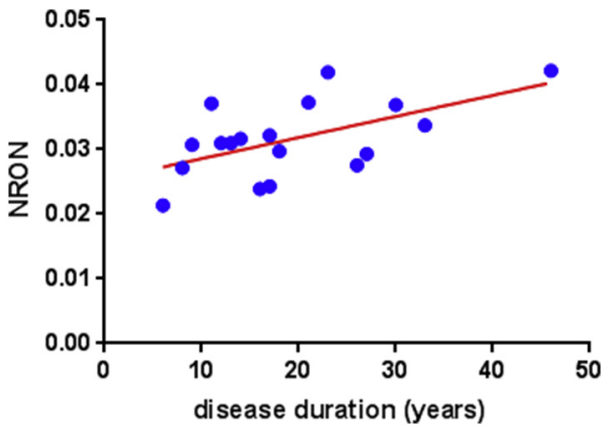

Fig. 6. Correlation between NRON levels and disease duration (years) in the replication cohort. $P=.048, R=0.478$. Correlation was calculated by Sperman's test.

lncRNAs in MS patients using a comprehensive technical approach. These findings globally support the hypothesis that dysregulations in lncRNAs expression levels could be relevant in MS pathogenesis. To date, the study of IncRNA is still in its infancy, although increasing evidence suggests their involvement in several pathogenic mechanisms in many disease, including MS. Moreover, several lncRNA have been identified, and each of them can regulate multiple processes, making difficult to identify potential lncRNA involved in MS basing merely on a candidate approach. Experience from extensive studies of miRNA in MS frequently yielded heterogeneous results, and have up to now not resulted in the identification of a blood-based biomarker for MS. This may be related to the fact that one miRNA can target many genes and the same gene can be regulated by many ncRNA, and that measurements have been done in different biologic fluids or cells. Therefore, further studies are needed to better clarify the usefulness of lncRNAs signature in clinical practice.

\section{Disclosure statement}

The authors have no actual or potential conflicts of interest.

\section{Ethic approval}

Written informed consent was obtained for all samples. All the experiments conformed with guidelines from the local Ethics Committee of IRCCS Fondazione Ca' Granda Ospedale Maggiore Policlinico.
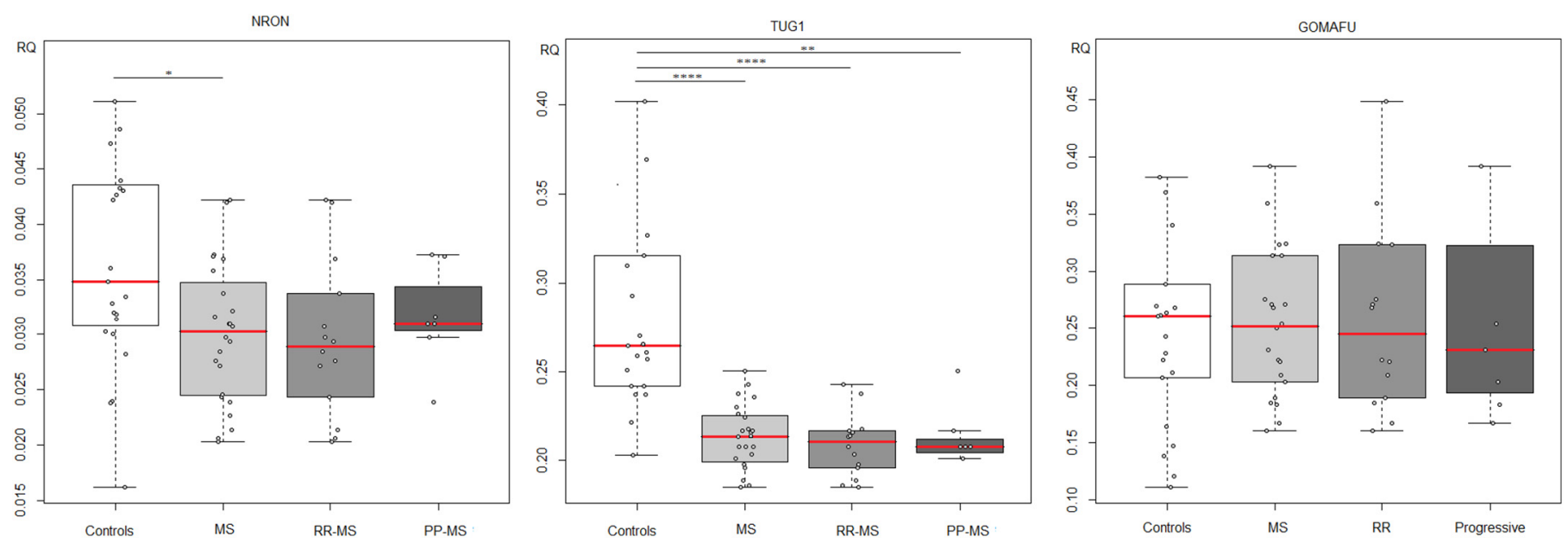

Fig. 5. Replication analysis. LncRNAs expresson levels obtained by ddPCR, in the Belgian cohort, are relative and expressed as relative quantification (RQ) (mean \pm SD) and stratified according the disease form. GAPDH and POLR2A were used as normalizer genes. Each dot represents one subject. Median of each group is indicated in red. Test of normality: Shapiro-Wilk's test. ${ }^{*} \mathrm{p}<.05,{ }^{* *} \mathrm{p}=.01{ }^{* * *} \mathrm{p}<.001{ }^{* * * *} \mathrm{p}<.0001$ calculated by non parametric Kruskall-Wallis test posthoc Dunn's test. (For interpretation of the references to colour in this figure legend, the reader is referred to the web version of this article.) 


\section{Consent for publication}

Not applicable.

\section{Availability of data and material}

The datasets used and/or analyzed during the current study are available from the corresponding author on reasonable request.

\section{Competing interests}

The authors declare that they have no competing interests.

\section{Funding}

This work was supported by grants from the Italian Ministry of Health. BD is a Clinical Investigator of the Research Foundation Flanders. AG and BD are supported by the Research CouncilKU Leuven (C24/16/045) and the Research Foundation Flanders (G.0734.15).

\section{Authors' contributions}

FC analyzed and interpreted the patient data regarding the discovery and validation cohort and contributed in writing the manuscript. EO performed replication analysis and contributed in writing the manuscript, MS, SMGC, MD, MA, contributed to process samples, MDR, AP, AC, EL, contributed to select and follow patients from the discovery cohort, CC, RC select the validation cohort, KM performed experiments in droplet digital PCR on the Belgian cohort, BD, AG analyzed the Belgian cohort data and revised the manuscript, ES and DG revised the manuscript.

\section{Acknowledgements}

Not applicable.

\section{References}

Aune, T.M., Crooke, P.S., Spurlock, C.F., 2016. Long noncoding RNAs in T lymphocytes. J. Leukoc. Biol. 99, 31-44 https://doi.org/10.1189/jlb.1RI0815-389R.

Barry, G., Briggs, J.A., Vanichkina, D.P., Poth, E.M., Beveridge, N.J., Ratnu, V.S., Nayler, S.P., Nones, K., Hu, J., Bredy, T.W., Nakagawa, S., Rigo, F., Taft, R.J., Cairns, M.J., Blackshaw, S., Wolvetang, E.J., Mattick, J.S., 2014. The long non-coding RNA Gomafu is acutely regulated in response to neuronal activation and involved in schizophrenia-associated alternative splicing. Mol. Psychiatry 19, 486-494. https:// doi.org/10.1038/mp.2013.45.

Dietz, L., Frommer, F., Vogel, A.-L., Vaeth, M., Serfling, E., Waisman, A., Buttmann, M., Berberich-Siebelt, F., 2015. NFAT1 deficit and NFAT2 deficit attenuate EAE via different mechanisms. Eur. J. Immunol. 45, 1377-1389. https://doi.org/10.1002/eji. 201444638.

Eftekharian, M.M., Ghafouri-Fard, S., Soudyab, M., Omrani, M.D., Rahimi, M., Sayad, A., Komaki, A., Mazdeh, M., Taheri, M., 2017. Expression Analysis of Long Non-coding RNAs in the Blood of Multiple Sclerosis patients. J. Mol. Neurosci. https://doi.org/10. 1007/s12031-017-0982-1.

Fenoglio, C., Ridolfi, E., Cantoni, C., De Riz, M., Bonsi, R., Serpente, M., Villa, C., Pietroboni, A.M., Naismith, R.T., Alvarez, E., Parks, B.J., Bresolin, N., Cross, A.H., Piccio, L.M., Galimberti, D., Scarpini, E., 2013. Decreased circulating miRNA levels in patients with primary progressive multiple sclerosis. Mult. Scler. J. 19, 1938-1942. https://doi.org/10.1177/1352458513485654.

Gandhi, R., Healy, B., Gholipour, T., Egorova, S., Musallam, A., Hussain, M.S., Nejad, P., Patel, B., Hei, H., Khoury, S., Quintana, F., Kivisakk, P., Chitnis, T., Weiner, H.L., 2013. Circulating MicroRNAs as biomarkers for disease staging in multiple sclerosis. Ann. Neurol. 73, 729-740. https://doi.org/10.1002/ana.23880.

Guttman, M., Donaghey, J., Carey, B.W., Garber, M., Grenier, J.K., Munson, G., Young, G., Lucas, A.B., Ach, R., Bruhn, L., Yang, X., Amit, I., Meissner, A., Regev, A., Rinn, J.L., Root, D.E., Lander, E.S., 2011. lincRNAs act in the circuitry controlling pluripotency and differentiation. Nature 477, 295-300. https://doi.org/10.1038/nature10398.

Johnson, R., 2012. Long non-coding RNAs in Huntington's disease neurodegeneration.
Neurobiol. Dis. 46, 245-254. https://doi.org/10.1016/j.nbd.2011.12.006

Khalil, A.M., Guttman, M., Huarte, M., Garber, M., Raj, A., Rivea Morales, D., Thomas, K., Presser, A., Bernstein, B.E., van Oudenaarden, A., Regev, A., Lander, E.S., Rinn, J.L., 2009. Many human large intergenic noncoding RNAs associate with chromatinmodifying complexes and affect gene expression. Proc. Natl. Acad. Sci. 106, 11667-11672. https://doi.org/10.1073/pnas.0904715106.

Kipanyula, M.J., Kimaro, W.H., Seke Etet, P.F., 2016. The emerging roles of the calcineurin-Nuclear factor of activated T-lymphocytes pathway in nervous System functions and diseases. J. Aging Res. 2016https://doi.org/10.1155/2016/5081021. 5081021.

Kleinewietfeld, M., Hafler, D.A., 2014. Regulatory T cells in autoimmune neuroinflammation. Immunol. Rev. 259, 231-244. https://doi.org/10.1111/imr.12169.

Lourenco, G.F., Janitz, M., Huang, Y., Halliday, G.M., 2015. Long noncoding RNAs in TDP-43 and FUS/TLS-related frontotemporal lobar degeneration (FTLD). Neurobiol. Dis. 82, 445-454. https://doi.org/10.1016/j.nbd.2015.07.011.

McFarland, H.F., Martin, R., 2007. Multiple sclerosis: a complicated picture of autoimmunity. Nat. Immunol. 8, 913-919. https://doi.org/10.1038/ni1507.

Mercer, T.R., Dinger, M.E., Sunkin, S.M., Mehler, M.F., Mattick, J.S., 2008. Specific expression of long noncoding RNAs in the mouse brain. Proc. Natl. Acad. Sci. U. S. A 105, 716-721. https://doi.org/10.1073/pnas.0706729105.

Mercer, T.R., Qureshi, I.A., Gokhan, S., Dinger, M.E., Li, G., Mattick, J.S., Mehler, M.F., 2010. Long noncoding RNAs in neuronal-glial fate specification and oligodendrocyte lineage maturation. BMC Neurosci. 11, 14. https://doi.org/10.1186/1471-2202$11-14$

Pahlevan Kakhki, M., Nikravesh, A., Shirvani Farsani, Z., Sahraian, M.A., Behmanesh, M., 2017. HOTAIR but not ANRIL long non-coding RNA contributes to the pathogenesis of multiple sclerosis. Immunology. https://doi.org/10.1111/imm.12850.

Polman, C.H., Reingold, S.C., Banwell, B., Clanet, M., Cohen, J.A., Filippi, M., Fujihara, K., Havrdova, E., Hutchinson, M., Kappos, L., Lublin, F.D., Montalban, X., O'Connor, P., Sandberg-Wollheim, M., Thompson, A.J., Waubant, E., Weinshenker, B., Wolinsky, J.S., 2011. Diagnostic criteria for multiple sclerosis: 2010 Revisions to the McDonald criteria. Ann. Neurol. 69, 292-302. https://doi.org/10.1002/ana.22366.

Rossi, S., Motta, C., Studer, V., Macchiarulo, G., Volpe, E., Barbieri, F., Ruocco, G., Buttari, F., Finardi, A., Mancino, R., Weiss, S., Battistini, L., Martino, G., Furlan, R., Drulovic, J., Centonze, D., 2014. Interleukin-1 $\beta$ causes excitotoxic neurodegeneration and multiple sclerosis disease progression by activating the apoptotic protein p53. Mol. Neurodegener. 9, 56. https://doi.org/10.1186/1750-1326-9-56.

Santoro, M., Nociti, V., Lucchini, M., De Fino, C., Losavio, F.A., Mirabella, M., 2016. Expression Profile of Long Non-Coding RNAs in Serum of patients with Multiple Sclerosis. J. Mol. Neurosci. 59, 18-23. https://doi.org/10.1007/s12031-016-0741-8.

Shi, X., Sun, M., Liu, H., Yao, Y., Song, Y., 2013. Long non-coding RNAs: a new frontier in the study of human diseases. Cancer Lett. 339, 159-166. https://doi.org/10.1016/j. canlet.2013.06.013.

Sinha, S., Boyden, A.W., Itani, F.R., Crawford, M.P., Karandikar, N.J., 2015. CD8(+) TCells as Immune regulators of Multiple Sclerosis. Front. Immunol. 6 (619). https:// doi.org/10.3389/fimmu.2015.00619.

Sun, D., Yu, Z., Fang, X., Liu, M., Pu, Y., Shao, Q., Wang, D., Zhao, X., Huang, A., Xiang, Z., Zhao, C., Franklin, R.J., Cao, L., He, C., 2017. LncRNA GAS5 inhibits microglial M2 polarization and exacerbates demyelination. EMBO Rep. 18, 1801-1816. https:// doi.org/10.15252/embr.201643668.

Trapp, B.D., Nave, K.-A., 2008. Multiple sclerosis: an immune or neurodegenerative disorder? Annu. Rev. Neurosci. 31, 247-269. https://doi.org/10.1146/annurev. neuro.30.051606.094313.

Tsuiji, H., Yoshimoto, R., Hasegawa, Y., Furuno, M., Yoshida, M., Nakagawa, S., 2011. Competition between a noncoding exon and introns: Gomafu contains tandem UACUAAC repeats and associates with splicing factor-1. Genes Cells 16, 479-490. https://doi.org/10.1111/j.1365-2443.2011.01502.x.

Turner, M., Galloway, A., Vigorito, E., 2014. Noncoding RNA and its associated proteins as regulatory elements of the immune system. Nat. Immunol. 15, 484-491. https:// doi.org/10.1038/ni.2887.

Wan, P., Su, W., Zhuo, Y., 2017. The Role of Long Noncoding RNAs in Neurodegenerative Diseases. Mol. Neurobiol. 54, 2012-2021. https://doi.org/10.1007/s12035-0169793-6.

Wu, P., Zuo, X., Deng, H., Liu, X., Liu, L., Ji, A., 2013. Roles of long noncoding RNAs in brain development, functional diversification and neurodegenerative diseases. Brain Res. Bull. 97, 69-80. https://doi.org/10.1016/j.brainresbull.2013.06.001.

Wu, G.-C., Pan, H.-F., Leng, R.-X., Wang, D.-G., Li, X.-P., Li, X.-M., Ye, D.-Q., 2015. Emerging role of long noncoding RNAs in autoimmune diseases. Autoimmun. Rev. 14, 798-805. https://doi.org/10.1016/j.autrev.2015.05.004.

Zhang, Z., Zhang, R., 2015. Epigenetics in autoimmune diseases: Pathogenesis and prospects for therapy. Autoimmun. Rev. 14, 854-863. https://doi.org/10.1016/j.autrev. 2015.05 .008$.

Zhang, F., Gao, C., Ma, X.-F., Peng, X.-L., Zhang, R.-X., Kong, D.-X., Simard, A.R., Hao, J.W., 2016. Expression Profile of Long Noncoding RNAs in Peripheral Blood Mononuclear Cells from Multiple Sclerosis patients. CNS Neurosci. Ther. 22, 298-305. https://doi.org/10.1111/cns.12498.

Zhou, X., Xu, J., 2015. Identification of Alzheimer's disease-associated long noncoding RNAs. Neurobiol. Aging 36, 2925-2931. https://doi.org/10.1016/j.neurobiolaging. 2015.07.015. 